\title{
Prevalence of the Polar Vessel Sign in Parathyroid Adenomas on the Arterial Phase of 4D CT
}

\author{
M. Bahl, M. Muzaffar, G. Vij, J.A. Sosa, K. Roy Choudhury, and J.K. Hoang
}

\begin{abstract}
BACKGROUND AND PURPOSE: The "polar vessel" sign has been previously described on sonography of parathyroid adenomas. We estimated the 4D CT prevalence of the polar vessel sign and determined features of parathyroid adenomas that are associated with this sign.
\end{abstract}

MATERIALS AND METHODS: Twenty-eight consecutive patients with parathyroid adenomas underwent 4D CT between 2008 and 2012 at 2 institutions. 4D CT images were reviewed for the presence of the polar vessel sign and a second vascular finding of an enlarged ipsilateral inferior thyroid artery. The polar vessel sign was correlated with adenoma weight and size and arterial phase CT attenuation.

RESULTS: Thirty-two parathyroid adenomas in 28 patients were studied, with a mean adenoma weight of $0.66 \pm 0.65 \mathrm{~g}$, a mean maximal CT diameter of $11.1 \pm 4.9 \mathrm{~mm}$, and a mean arterial attenuation of $148 \pm 47 \mathrm{HU}$. The polar vessel sign was seen in 20/32 (63\%) adenomas. Adenomas with a polar vessel had higher arterial phase attenuation than adenomas without a polar vessel $(163$ and $122 \mathrm{HU}$, respectively, $P<$ .01). Size and weight were not significantly different for adenomas with and without polar vessels. An enlarged inferior thyroid artery was seen in only $2 / 28(7 \%)$ patients with unilateral disease.

CONCLUSIONS: The polar vessel sign was present in nearly two-thirds of parathyroid adenomas on 4D CT and was more likely to be present in adenomas that had greater arterial phase enhancement. This sign can be used along with enhancement characteristics to increase the radiologist's confidence that a visualized lesion is a parathyroid adenoma rather than a thyroid nodule or lymph node.

4 D parathyroid CT is increasingly used for preoperative localization of parathyroid adenomas. 4D CT was first described in 2006 and involves $\geq 2$ contrast phases with high-resolution multiplanar images of the neck and upper chest. ${ }^{1}$ The characteristic features of parathyroid adenomas on 4D CT are low attenuation on noncontrast imaging, peak enhancement on the arterial phase, and washout of contrast on the venous phase. ${ }^{2-4}$ These allow differentiation between a parathyroid adenoma and its mimics, such as thyroid nodules and lymph nodes.

Characteristic enhancement may not be evident in all cases due to adenoma composition, small adenoma size, or suboptimal arterial timing. In these cases, the radiologist must rely on his or her knowledge of the expected locations of adenomas and morphologic findings. A morphologic feature that has been previously

Received May 19, 2013; accepted after revision June 21.

From the Departments of Radiology (M.B., G.V., K.R.C., J.K.H.), Surgery, Division of Surgical Oncology (J.A.S.), and Radiation Oncology (J.K.H.), Duke University Medical Center, Durham, North Carolina; and Department of Radiology (M.M.), Carle Foundation Hospital, Urbana, Illinois.

Please address correspondence to Jenny K. Hoang, MD, Department of Radiology, Duke University Medical Center, Box 3808, Erwin Rd, Durham, NC 27710; e-mail: jennykh@gmail.com

http://dx.doi.org/10.3174/ajnr.A3715

described on sonography is a feeding artery (usually a branch of the inferior thyroid artery) to the adenoma. ${ }^{5,6}$ Small arteries cannot be seen on nuclear scintigraphy or MR imaging because of lower spatial resolution, and they have not been described on single venous phase $\mathrm{CT}$, to our knowledge. In a recent review article, the authors noted the presence of a "polar vessel" sign on the arterial phase of $4 \mathrm{D} \mathrm{CT}{ }^{4}$ The polar vessel sign can represent either an enlarged feeding artery or a draining vein seen on the arterial phase of imaging, and it is characterized by a vessel that terminates in a parathyroid adenoma and that is larger than contralateral neck vessels in a similar location.

The purpose of this study was to estimate the prevalence of the polar vessel sign on 4D CT and to determine features of parathyroid adenomas that are associated with this sign. We hypothesize that a polar vessel may be more commonly seen in adenomas that are larger or have more vivid arterial enhancement.

\section{MATERIALS AND METHODS Subjects}

This was a retrospective study, from 2 institutions, of patients with primary hyperparathyroidism who underwent 4D CT between January 2008 and December 2012 as a localization study before parathyroid surgery. The study was approved by the institutional review 
board at both institutions and was compliant with the Health Insurance Portability and Accountability Act. We identified patients at both institutions by performing a radiology data base search of patients who had undergone 4D CT before surgery. Most 4D CT scans were ordered when sonography and scintigraphy findings were discordant or negative; but in 6 cases, 4D CT was the first investigation. Patients were excluded if the arterial phase scan was poorly timed (venous attenuation greater than arterial attenuation) because the polar vessel sign is only seen on arterial phase imaging. There were 34 patients with parathyroid adenomas. Six of the 34 were excluded due to the absence of optimal arterial phase imaging, with excluded cases more common in the first year of imaging due to development of the protocols.

Hospital electronic medical records were accessed to obtain the following information for each subject: basic demographics, operative reports, and surgical pathology reports.

\section{D CT Imaging Technique}

4D CT scans at our institutions were obtained on different scanner types with different protocols. Studies at Duke University Medical Center were performed on 64-row multidetector CT scanners (750 HD and LightSpeed; GE Healthcare, Milwaukee, Wisconsin), and studies at Carle Foundation Hospital were performed on a 16-row multidetector CT scanner (LightSpeed; GE Healthcare). All CT scans included at least arterial and venous phase CT.

The current protocol at Duke University Medical Center consists of 3 imaging phases. The first phase is a noncontrast CT to include the thyroid gland; the z-axis is from the hyoid bone to the clavicular head. The next 2 phases are contrast phases from the angle of the mandible to the carina and are performed after intravenous administration of $75 \mathrm{~mL}$ of iopamidol (Isovue-300; Bracco, Princeton, New Jersey) via a 20-ga cannula in a right antecubital vein at a rate of $4 \mathrm{~mL} / \mathrm{s}$, followed by a $25-\mathrm{mL}$ saline chaser. Arterial phase images are acquired 25 seconds after the start of the injection, and the delayed (venous) phase images are acquired 80 seconds from the start of the injection. The parameters for all 3 phases are the following: 0.625-mm section thickness; tube rotation time, 0.4 seconds; pitch factor, 0.516:1; FOV, $20 \mathrm{~cm} ; 120 \mathrm{kV}$ (peak); and automatic tube current modulation (Smart mA, GE Healthcare; with noise index, 10; minimum $100 \mathrm{~mA}$ and maximum 700 $\mathrm{mA}$ ). One-millimeter-thick contiguous axial images in all 3 phases are sent to the PACS for interpretation and to allow manipulation of images on a 3D workstation. Reformatted images in the arterial phases are sent to the PACS as 2.5-mm-thick contiguous images in the axial, coronal, and sagittal planes.

CT scans at Carle Foundation Hospital consist of arterial and venous phases from the angle of the mandible to the carina and are obtained after intravenous administration of $100 \mathrm{~mL}$ of iopamidol (Isovue-300) via a 20-ga cannula in the right antecubital vein at a rate of $4 \mathrm{~mL} / \mathrm{s}$, followed by a $50-\mathrm{mL}$ saline chaser. The arterial phase is acquired 20 seconds after the start of the injection, and the delayed (venous) phase is acquired 70 seconds from the start of the injection. The parameters for both phases are the following: 0.625-mm section thickness; tube rotation time, $0.5 \mathrm{sec}-$ onds; pitch factor, 1.75:1, FOV, $20 \mathrm{~cm} ; 120 \mathrm{kVp}$; and automatic tube current modulation (Smart $\mathrm{mA}$ with noise index 11; minimum $100 \mathrm{~mA}$ and maximum $440 \mathrm{~mA}$ ). For interpretation, 1.25-mm reconstructed contiguous axial images and 1-mm-thick sagittal and coronal reformatted images in arterial and venous phases are sent to the PACS.

\section{Image Interpretation}

4D CT images were reviewed on a PACS workstation. Readers were an attending neuroradiologist, a neuroradiology fellow, and a diagnostic radiology resident with 11,5 , and 2 years of CT experience, respectively. Readers were given information about the location of the parathyroid adenoma based on the surgical findings and were asked to evaluate the CT scans for the presence of the polar vessel sign and a second vascular finding of an enlarged ipsilateral inferior thyroid artery on the arterial phase images. Readers were not assessed on their ability to detect the parathyroid adenoma because this was not a study on the performance of $4 \mathrm{D}$ CT for localization. The 2 junior readers were educated on how to recognize the vascular findings before starting the study. The polar vessel sign usually represents an enlarged feeding artery; but when followed to the origin, it can also represent a draining vein. It is characterized by a vessel that terminates in the parathyroid adenoma and is usually larger than contralateral neck vessels in a similar location (Figs 1 and 2). The polar vessel can be tortuous (Fig 1). An enlarged inferior thyroid artery was defined as increased enhancement or size of the artery on the side of the parathyroid adenoma compared with the artery in the contralateral neck (Fig 3). This was best assessed on axial and coronal images.

Note that Figs 1-3 are maximum-intensity-projection reconstructions to display the polar vessel on a single image. The images were not reviewed as maximum-intensity-projection images by the readers in the study or in the clinical setting. The polar vessel sign was assessed for all adenomas. The enlarged ipsilateral inferior thyroid artery was only evaluated in cases with unilateral disease (ie, not cases of bilateral multiglandular disease). The readers were not blinded to the location and number of parathyroid adenomas because the purpose of the study was not to determine the accuracy of 4D CT.

Other characteristics of the parathyroid adenomas, including location, dimensions, and arterial phase imaging attenuation, were also recorded. On arterial phase imaging, the mean attenuation was calculated on the basis of 3 region-of-interest measurements within the center of the parathyroid adenoma, avoiding cystic areas.

\section{Data Analysis}

The data were entered into an Excel spreadsheet (2007 Version; Microsoft, Redmond, Washington). Statistical analyses were performed by using SAS Enterprise (Version 4.2; SAS Institute, Cary, North Carolina) and the R package (www.r-project.org). Two-sample $t$ tests were used to compare features of parathyroid adenomas with and without a polar vessel. The Fleiss $\kappa$ was calculated to determine interrater agreement for the presence of a polar vessel and an enlarged ipsilateral thyroid artery. Agreement was regarded as poor with Fleiss $\kappa \leq 0.20$, as slight with $0.21-0.40$, as moderate with $0.41-$ 0.60 , as substantial with $0.61-0.80$, and as almost perfect with $0.81-$ $1.00{ }^{7} P$ values $<.05$ were considered statistically significant.

\section{RESULTS}

\section{Characteristics of Patients and Parathyroid Adenomas}

The study population comprised 28 patients ( 24 females; mean age, 60 years; range, $15-83$ years). All patients had parathyroid

AJNR Am J Neuroradiol 35:578-81 Mar 2014 www.ajnr.org 


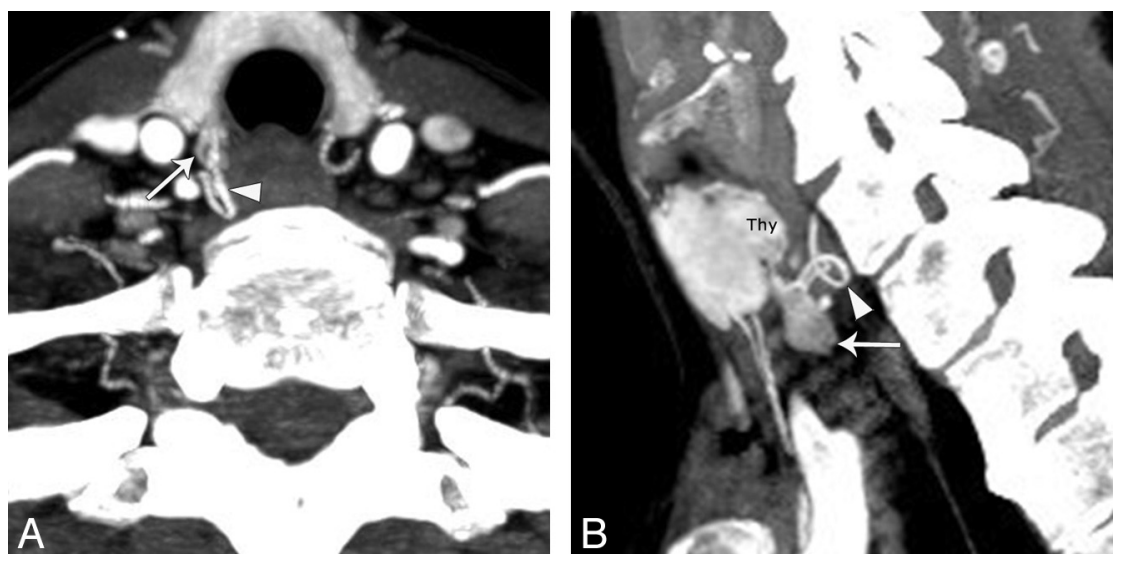

FIG 1. A 69-year-old woman with a right parathyroid adenoma. Axial $(A)$ and sagittal $(B)$ arterial phase images demonstrate an oval-shaped hyperenhancing adenoma posterior and inferior to the lower pole of the right thyroid gland (straight arrows). A characteristic tortuous feeding artery is seen at the superior aspect of the adenoma (arrowheads).

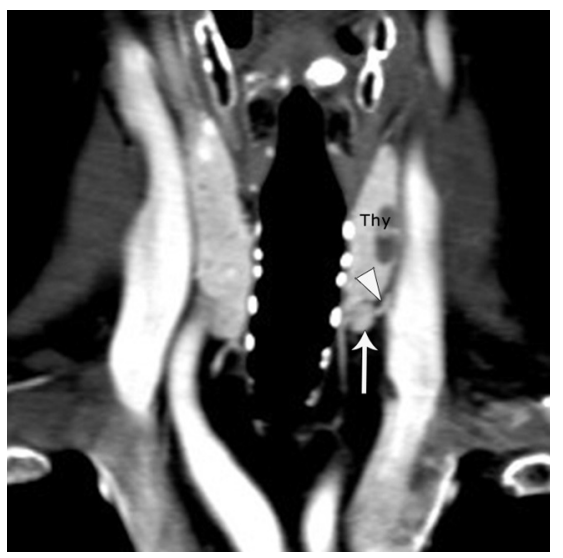

FIG 2. A 71-year-old woman with a left parathyroid adenoma. Coronal arterial phase image demonstrates a round hyperenhancing adenoma inferior to the lower pole of the left thyroid gland (straight arrow). A characteristic feeding artery (arrowhead) is seen at the superolateral aspect of the adenoma.

adenomas that could be identified on 4D CT in retrospect. Twenty-four of 32 (75\%) parathyroid adenomas were reported prospectively in the radiology report. These cases represented the initial 4D CT scans at both institutions after introducing the protocol, and accuracy of localization was likely improved with experience. Four of 28 (14\%) patients had double adenomas, and 2 of 28 (7\%) had adenomas in ectopic locations (retropharynx and carotid space). The 32 adenomas in 28 patients had a mean weight of $0.66 \pm 0.65 \mathrm{~g}$, a mean maximal CT diameter of $11.1 \pm 4.9 \mathrm{~mm}$, and a mean arterial attenuation of $148 \pm 47 \mathrm{HU}$.

\section{Vascular Findings}

The polar vessel sign was present in 23 (72\%), 23 (72\%), and 24 (75\%) adenomas according to readers A, B, and C, respectively. All 3 readers agreed on the presence of a polar vessel sign in 20 of $32(63 \%)$ adenomas. At least 2 of 3 readers agreed on the presence of a polar vessel sign in 24 of $32(75 \%)$ adenomas. The Fleiss $\kappa$ (an index of interrater agreement) was 0.68 (95\% CI, 0.49-0.84), which indicates substantial agreement.

\section{DISCUSSION} parathyroid adenoma."
An enlarged inferior thyroid artery was present in $2(7 \%), 6(21 \%)$, and 3 (11\%) adenomas according to readers A, B, and C, respectively. All 3 readers agreed on the presence of an enlarged inferior thyroid artery in 2 of $28(7 \%)$ patients with unilateral disease. At least 2 of 3 readers agreed on the presence of an enlarged inferior thyroid artery in 3 of $28(11 \%)$ patients. The Fleiss $\kappa$ was 0.58 (95\% CI, -0.04-0.84), which indicates moderate agreement.

\section{Polar Vessel Sign and Parathyroid Adenoma Characteristics}

For this analysis, an adenoma was considered to have a polar vessel only if all 3 readers agreed on its presence. Adenomas with a polar vessel had statistically significant higher arterial phase attenuation than adenomas without a polar vessel (163 and $122 \mathrm{HU}$, respectively; $P<.01)$. Adenomas with a polar vessel trended toward higher mean maximum diameters than adenomas without a polar vessel (12.4 and $9.0 \mathrm{~mm}$, respectively), but this difference was not statistically significant $(P=.07)$. There were no differences in gross pathologic weights of adenomas with or without the polar vessel $(0.62$ and $0.80 \mathrm{~g}$, respectively; $P=.6)$.

We report the prevalence of the polar vessel sign on parathyroid $4 \mathrm{D} \mathrm{CT}$, as well as the features of parathyroid adenomas associated with this sign. We found the polar vessel sign to be present in nearly two-thirds of parathyroid adenomas on $4 \mathrm{D}$ CT and to be more common in adenomas with greater arterial phase enhancement. The sign is common and can be used along with enhancement characteristics to increase the radiologist's confidence that a visualized lesion is a parathyroid adenoma.

Parathyroid adenomas can be difficult to differentiate from thyroid nodules, level VI lymph nodes, or ectopic thyroid tissue. Unlike parathyroid adenomas, lymph nodes and thyroid nodules are less likely to have prominent feeding arteries or draining veins that are visible on CT. We found the polar vessel sign to be confidently seen on $4 \mathrm{D}$ CT in $63 \%$ of adenomas by all readers and up to $75 \%$ of adenomas by 2 readers. Therefore, this sign can be regarded as common and useful. The polar vessel alone is not diagnostic of a parathyroid adenoma. In our clinical practice, the other signs of parathyroid adenoma, such as shape and enhancement, are used primarily to identify them. The presence of the polar vessel sign does, however, increase the confidence that the lesion is a parathyroid adenoma and may change the interpretation of "suspicious for parathyroid adenoma" to "in keeping with

Our results are in accordance with findings of a polar vessel in the sonography literature. Lane et $\mathrm{al}^{6}$ reported sonographic findings of an extrathyroidal artery leading to a parathyroid adenoma in 35 of $42(83 \%)$ adenomas and found that the presence of an extrathyroidal artery aided in the detection of an otherwise inconspicuous parathyroid gland in several patients. Wolf et $\mathrm{al}^{5}$ identi- 


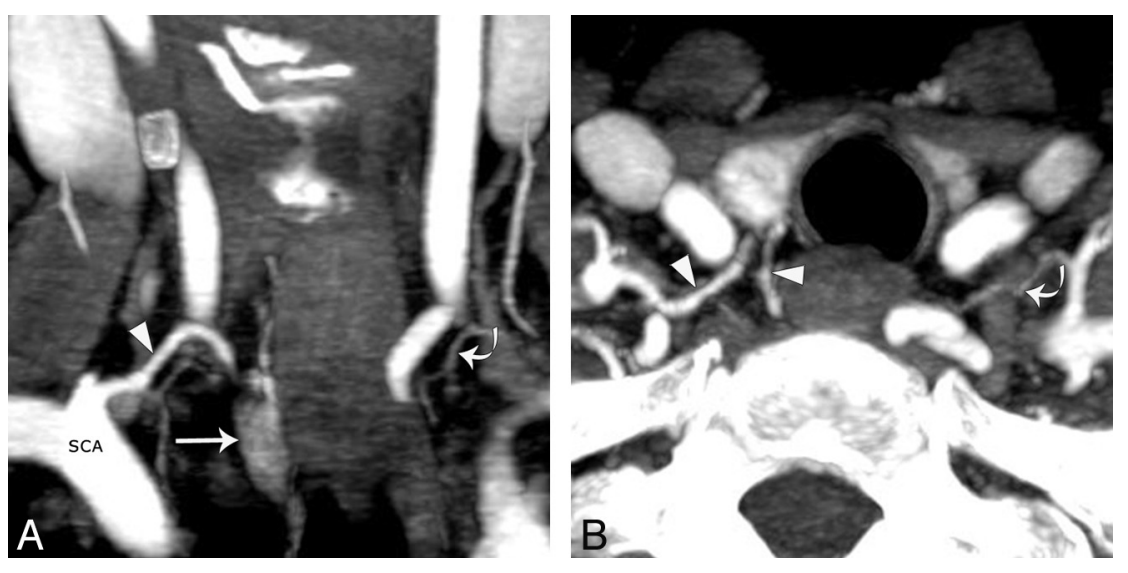

FIG 3. A 70-year-old woman with a right parathyroid adenoma. A, Coronal arterial phase image demonstrates an oval hyperenhancing adenoma in the right tracheoesophageal groove (straight arrow). Coronal $(A)$ and axial $(B)$ images show an enlarged inferior thyroid artery (arrowheads) arising from the right subclavian artery and terminating at the superior pole of the adenoma. Note the normal contralateral inferior thyroid artery (curved arrows).

fied the presence of a demonstrable blood supply from the inferior thyroid artery branches as a vascular arc surrounding the gland from $90^{\circ}$ to $270^{\circ}$; in their series, 20 of $32(63 \%)$ parathyroid adenomas had a color arc. Scheiner et $\mathrm{al}^{8}$ reported finding a vessel draping over an adenoma in $65 \%$ of cases. The arterial phase of $4 \mathrm{D}$ CT allows radiologists to see the CT correlate of this well-established sonographic vascular finding.

We attempted to identify adenomas that were more likely to have the polar vessel sign. The only characteristic that was significantly more likely to have the polar vessel sign was higher arterial phase attenuation. Higher CT attenuation or enhancement is a marker of the blood supply of a tissue. Hypersecreting parathyroid glands have increased blood supply. ${ }^{6}$ In fact, periadenomal vessels were described in the angiographic literature in the 1980s and accounted for the early experience with angiographic ablation for the treatment of parathyroid adenomas. ${ }^{9,10}$ These vessels are theorized to be enlarged normal vessels, parasitized by the parathyroid adenoma. ${ }^{10}$ Thus, adenomas that are more vascular and enhance more on CT are more likely to have a visible polar vessel. An alternate explanation for the polar vessel sign being present in adenomas with higher arterial attenuation is related to the CT technique; specifically, a CT scan with a more optimally timed arterial phase would be more likely to show higher arterial phase enhancement of a parathyroid adenoma along with its associated vessel.

There are several limitations to our study. The major limitation is that the radiologists reading the scans knew that the adenomas were present and therefore might be more likely to report the presence of a polar vessel. The alternative of asking the readers to find the adenoma and then the polar vessel sign would make the study significantly more complicated, however, because we would be assessing the accuracy of $4 \mathrm{D}$ CT for localization and the presence of the sign. This assessment could lead to an underestimation of the sensitivity of 4D CT because only 1 radiologist has the clinical experience to interpret $4 \mathrm{D} \mathrm{CT}$ scans at the primary study institution. We thought readers of all levels were capable of reporting the presence or absence of a polar vessel sign in a known adenoma; thus, this study focuses on the prevalence of the polar vessel sign rather than its specificity and sensitivity for diagnosing parathyroid adenoma. We agree that in future studies, readers should be blinded to the surgical results, and this will be the focus of our future work.

Second, this was a retrospective clinical series with a small study population. Small study size may affect the estimate of the prevalence of vascular findings and result in type II errors for the polar vessel associations, but it is reassuring to see that our prevalence estimates are similar to those in the sonography literature. Third, the CT scans were from 2 different institutions and were obtained with different protocols during the study period. The variation in scanning technique should not affect the data collected for adenoma diameter and arterial phase attenuation because all patients had arterial phase CT imaging.

\section{CONCLUSIONS}

The polar vessel sign is present in nearly two-thirds of surgically confirmed parathyroid adenomas on 4D CT and is more likely to be present in adenomas that have greater arterial phase enhancement. This sign may be used along with enhancement characteristics to potentially increase the radiologist's confidence that a visualized lesion is a parathyroid adenoma rather than a thyroid nodule or lymph node.

\section{REFERENCES}

1. Rodgers SE, Hunter GJ, Hamberg LM, et al. Improved preoperative planning for directed parathyroidectomy with 4-dimensional computed tomography. Surgery 2006;140:932-40, discussion 940-41

2. Beland MD, Mayo-Smith WW, Grand DJ, et al. Dynamic MDCT for localization of occult parathyroid adenomas in 26 patients with primary hyperparathyroidism. AJR Am J Roentgenol 2011;196:61-65

3. Gafton AR, Glastonbury CM, Eastwood JD, et al. Parathyroid lesions: characterization with dual-phase arterial and venous enhanced CT of the neck. AJNR Am J Neuroradiol 2012;33:949-52

4. Hoang JK, Sung WK, Bahl M, et al. How to do parathyroid 4D-CT: tips and traps for technique and interpretation. Radiology 2014;270:15-24

5. Wolf RJ, Cronan JJ, Monchik JM. Color Doppler sonography: an adjunctive technique in assessment of parathyroid adenomas. $\mathrm{J} \mathrm{Ul}$ trasound Med 1994;13:303-08

6. Lane MJ, Desser TS, Weigel RJ, et al. Use of color and power Doppler sonography to identify feeding arteries associated with parathyroid adenomas. AJR Am J Roentgenol 1998;171:819-23

7. Landis JR, Koch GG. The measurement of observer agreement for categorical data. Biometrics 1977;33:159-74

8. Scheiner JD, Dupuy DE, Monchik JM, et al. Pre-operative localization of parathyroid adenomas: a comparison of power and colour Doppler ultrasonography with nuclear medicine scintigraphy. Clin Radiol 2001;56:984-88

9. Miller DL, Doppman JL, Chang R, et al. Angiographic ablation of parathyroid adenomas: lessons from a 10-year experience. Radiology 1987;165:601-07

10. Doppman JL, Brennan MF, Kahn CR, et al. Circumscribing or periadenomal vessel: a helpful angiographic finding in certain islet cell and parathyroid adenomas. AJR Am J Roentgenol 1981; 136:163-65 